MINI-SYMPOSIUM

\title{
It's a matter of time: contemporary pre-hospital management of acute ST elevation myocardial infarction
}

\author{
R C Welsh, P W Armstrong
}

Heart 2005;91:1524-1526. doi: 10.1136/hrt.2004.055616

S ubstantial intellectual and financial resources have been invested in the quest to reduce outcomes in patients with ST elevation myocardial infarction (STEMI) through improved pharmacological and mechanical reperfusion therapies. By contrast, little emphasis has been placed on enhancing the medical systems whereby these treatments are delivered, especially as it relates to pre-hospital reperfusion. In this article we will review (1) the importance of timely reperfusion, (2) data supporting pre-hospital fibrinolysis, and (3) a novel contemporary systems based approach to improving outcomes in patients with acute cardiac events.

\section{THE IMPORTANCE OF TIME}

Delay in treatment is associated with a substantial reduction in the benefit of fibrinolysis as demonstrated in a metaanalysis of greater than 50000 patients with STEMI. ${ }^{1}$ Treatment within the first hour of symptoms achieves twice the benefit of subsequent treatment $(<1$ hour $=65 / 1000$ patients treated lives $v 1-2$ hours $=37$ lives $/ 000$ patients treated). This validated experimental models of acute myocardial infarction, which demonstrated a continuous and progressive wave of myocardial necrosis dependent on the duration of coronary artery ligation. ${ }^{2}$ These and other data spearheaded a focus on reducing time to reperfusion and spawned the adages "golden hour of fibrinolysis" and "time is muscle".

Although the negative impact of temporal delay has been a key feature of fibrinolysis research, substantial debate exists over whether this phenomenon applies equally to percutaneous coronary intervention (PCI). In the primary PCI arm of DANAMI-2 there was a threefold increase in events in patients randomised later compared to earlier $(1.5$ hours $=$ $4.7 \% \vee 4-12$ hours $=12.2 \% 30$ day death, re-myocardial infarction (MI), stroke). ${ }^{3}$ In GUSTO-2B the in-hospital delay from randomisation until first balloon inflation was associated with a sixfold increase in death comparing patients treated within the first 60 minutes and those treated after 90 minutes ( $1 \% \vee 6.4 \%$, 30 day mortality). ${ }^{4}$ Based on analysis of all randomised trials of fibrinolysis versus primary PCI, the mortality benefit of PCI was shown to decrease at a rate of $0.94 \%$ for every 10 minutes of delay. ${ }^{5}$ The two strategies become equivalent after 62 minutes from when fibrinolysis could have been administered in anticipation of primary PCI. Hence, this temporal target for reperfusion and the notion of a 60 minute maximally acceptable interval between strategies has been incorporated into the recent American College of Cardiology/American Heart Association STEMI guidelines as well as a Canadian perspective on these guidelines. ${ }^{67}$

In patients that present early in the course of STEMI excellent outcomes are achieved with fibrinolysis, at least equivalent or even superior to primary PCI. The PRAGUE-2 study demonstrated that within three hours of symptom onset, mechanical reperfusion was similar to streptokinase $\left(7.3 \% \vee 7.4 \%, 30\right.$ day mortality). ${ }^{8}$ In the CAPTIM trial patients treated pre-hospital within two hours of symptom onset had a 30 day mortality favouring fibrinolysis (accelerated tissue plasminogen activator (tPA)) (2.2\% $v 5.7 \%$, $\mathrm{p}=0.058)$ with an associated reduction in the rate of cardiogenic shock $(1.3 \% \quad v 5.3 \%, p=0.032) .{ }^{9}$ A large prospective French nationwide registry demonstrated that patients receiving pre-hospital fibrinolysis and admitted to hospital within 3.5 hours (median time) have no in-hospital mortality and one year survival of $99 \% .{ }^{10}$ Furthermore, it was recently demonstrated that $25 \%$ of patients treated with fibrinolysis within the first hour leave the hospital with no evidence of myocardial necrosis, a concept termed aborted myocardial infarction. ${ }^{11}$ In those patients that present to the medical system within three hours, the acceptable delay for mechanical reperfusion is even less than stated above and pharmacological reperfusion may represent the reperfusion strategy of choice.

\section{PRE-HOSPITAL FIBRINOLYSIS}

Time to treatment in major clinical STEMI trials remains stalled at approximately three hours after symptom onset and attempts to decrease delay through public and professional education have had limited impact. $^{12}{ }^{13}$ The concept of initiating pharmacological reperfusion in the pre-hospital setting is founded on a logical approach of utilising prehospital teams to decrease unnecessary delay and improve patient outcomes. Although international acceptance and implementation has been less than ideal, thought leaders in this arena have validated the concept over two decades of clinical experience and success. The recently published prospective French nationwide registry substantiates the benefit of fibrinolysis as delivered in a mature physician based pre-hospital system. ${ }^{10}$ In 443 participating centres, 1922 myocardial infarction patients (STEMI and non-STEMI) were identified of which 9\% (180/1922) received pre-hospital fibrinolysis, 19\% in-hospital fibrinolysis (365/1922), 23\% primary PCI (434/1922), and $49 \%$ no reperfusion therapy (943/1922), with in-hospital death rates of 3.3\%, 8.0\%, 6.7\%, and $12.2 \%$, respectively. At one year the benefit of prehospital fibrinolysis was maintained with 94\% survival compared to $89 \%$ in both the in-hospital fibrinolysis and primary PCI groups and a relative risk of death of 0.52 (95\% confidence interval (CI) 0.25 to $1.08 ; \mathrm{p}=0.08$ ).

An analysis of randomised trials of pre-hospital versus inhospital fibrinolysis has demonstrated a one hour reduction in time to treatment with substantial improvement in patient mortality ( $1.7 \%$ absolute reduction, odds ratio (OR) $0.83,95 \%$ CI 0.70 to 0.98$).{ }^{14}$ Contemporary trials have expanded this knowledge and addressed perceived limitations of past research and impediments to implementation of broad scale pre-hospital fibrinolysis. ${ }^{15-17}$ In the 12 countries participating in ASSENT 3+, substantial variability in population density,

Abbreviations: EMS, emergency medical system; MI, myocardial infarction; $\mathrm{PCl}$, percutaneous coronary intervention; STEMI, ST elevation myocardial infarction 


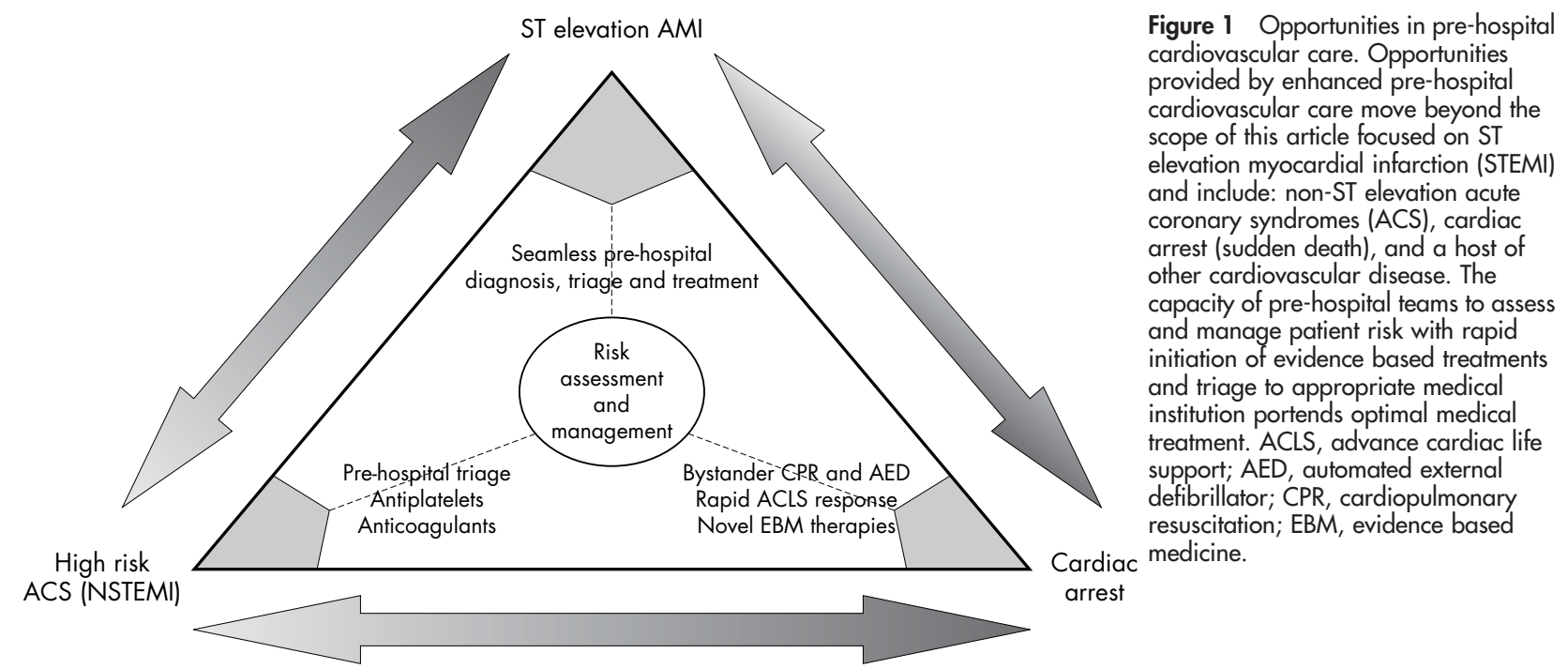

past experience with pre-hospital fibrinolysis ( $28 \%$ developed de novo programmes), and pre-hospital emergency medical service resources were demonstrated. ${ }^{18}$ In $60 \%$ of study sites a physician in the pre-hospital setting identified and assessed potential patients, interpreted the 12 lead ECG, obtained informed consent, and administrated fibrinolytic therapy. In the remainder of sites paramedical personnel completed onscene assessment, obtained and transmitted the 12 lead ECG for remote physician over-read, obtained informed consent, and administered fibrinolytic therapy. Preliminary analysis suggests that the presence of a physician in the pre-hospital setting was associated with greater adherence to protocol mandated treatments and procedures, but delay in time to treatment. ${ }^{18}{ }^{19}$ Although the clinical experience and enhanced training of a physician in the pre-hospital setting may represent the ideal situation; trials (MITI, ER-TIMI-19, and ASSENT 3+) and the wealth of clinical experience worldwide has demonstrated efficacy and safety of paramedic based prehospital fibrinolysis programmes. In the vast majority of health care systems worldwide the presence of a pre-hospital physician is impractical and unrealistic. Empowerment of adequately trained paramedical teams to administer lifesaving treatment such as fibrinolysis pre-hospital deserves, in our view, to constitute a high international priority.

Approximately half of STEMI patients activate the prehospital emergency medical system as the first contact for medical assistance. ${ }^{20}{ }^{21}$ In the two largest contemporary trials of pre-hospital fibrinolysis median time to treatment of two hours was achieved (CAPTIM and ASSENT 3+). ${ }^{16}{ }^{17}$ In contrast, median time to treatment with trials of in-hospital fibrinolysis, which includes patients that present by ambulance and of their own accord, remains locked closer to three hours and registry data documents even further delay. ${ }^{22}$ The fact that patients with STEMI who activate the pre-hospital emergency medical system can achieve rapid fibrinolysis, and that this in turn portends excellent clinical outcomes, equivalent or superior to primary PCI, provides powerful impetus for health care systems to implement such programmes.

\section{CONTEMPORARY SYSTEMS BASED APPROACH TO STEMI CARE}

Increasingly, arguments favouring one or other method of reperfusion are being supplanted by more strategic approaches to advancing optimal reperfusion therapy which depend on specific patient characteristics, time of presentation, as well as the local expertise and resources. Optimal care for patients that activate the emergency medical system (EMS) requires rapid EMS response, and patient assessment/ stabilisation with pre-hospital diagnosis and treatment. Assessment of risk related to patient characteristics, mode of reperfusion, and response to therapy allows seamless triage to the most appropriate hospital destination. In a large proportion of patients this should include administration of pre-hospital fibrinolysis. In the presence of contraindication to fibrinolysis or when primary PCI is consistently available within 60 minutes of diagnosis, direct triage to an experienced interventional centre with pre-hospital notification should minimise unnecessary delay. The knowledge and expertise required to safely triage and transport a patient for primary PCI is entirely complementary to that required for administration of pre-hospital fibrinolysis. Regardless of a region's emphasis on method of reperfusion, the pre-hospital teams responsible for assessment, stabilisation, treatment, triage and/or transport of this high risk patient population play a pivotal role. Furthermore, if facilitated PCI, where patients receive pharmacological reperfusion with urgent

Table 1 Optimal future components of pre-hospital cardiac care

\begin{tabular}{ll}
\hline & Resource \\
\hline Personnel & 2 ACLS trained paramedics available within 7 \\
Information & minutes of call \\
technology & Reliable communication with remote physician \\
& - Seamless electronic health record \\
& Online capacity to interrogate available \\
& resources (ED beds, CCU beds, cath lab \\
& capacity) \\
Pharmacological & - Current evidence based treatments: aspirin, \\
anticoagulation, bolus fibrinolysis, llb/llla \\
treatment
\end{tabular}

ACLS, advance cardiac life support; CCU, cardiac care unit; ED, emergency department; IABP, intra-aortic balloon pump. 
co-intervention, ultimately proves to be safe and effective, then pre-hospital fibrinolysis linked with angiography and PCI could provide optimal congruence between differing reperfusion therapies.

Although this article has focused on the management of STEMI patients, enhanced pre-hospital care establishes a platform from which to improve outcomes in a broad range of cardiovascular diseases including high risk acute coronary syndrome (non-STEMI) and cardiac arrest (fig 1). Cardiac arrest is associated with a very poor prognosis that can be substantially improved with early cardiopulmonary resuscitation, automated external defibrillators, rapid EMS response, and initiation of definitive treatment. ${ }^{23}$ Moreover, enhancing pre-hospital treatment platforms could facilitate incorporation of novel evidence based treatments, such as patient "cooling", early in the course of treatment. High risk acute coronary syndrome patients, identified through appropriate clinical, ECG, and other characteristics, would benefit from early administration of effective antiplatelet and anticoagulant treatment with triage to a centre capable of invasive investigation and management. Moving forward to optimise pre-hospital cardiac care resources will require the engagement of information technologies, optimal pharmacological and mechanical therapies, and the intellectual equity to implement proven treatments, especially those with important time dependency such as fibrinolysis in STEMI (table 1). The recognition of these capabilities has effectively redefined the point of first medical contact to the pre-hospital setting for many, and our future efforts should in part be directed towards increasing the proportion of patients that use this mode of access. There are now no logical reasons to delay response to the call to action; empowering the prehospital teams to administer effective evidence based treatment at the earliest point of contact.

The four main components of pre-hospital cardiac care are listed in table 1. Pre-hospital personnel, that may include a physician or adequately trained paramedical staff with appropriate remote physician support, consistently capable of rapid response to the aid of patients, forms the base for all interventions. Appropriate information technology facilitates care through: (1) communication, (2) seamless health record therefore minimising unnecessary repetition, and (3) assessment of the capacity of receiving hospitals. Effective administration of evidence based pharmacological and medical technologies at the earliest point of care portends optimal medical treatment.

\section{Authors' affiliations \\ R C Welsh, P W Armstrong, University of Alberta, Edmonton, Alberta,} Canada

Correspondence to: Dr Robert C Welsh, University of Alberta, 2C2.12, Walter Mackenzie Centre, 8440 - 112 Street, Edmonton, Alberta T6G 2B7, Canada; rwelsh@cha.ab.ca

\section{REFERENCES}

1 Boersma E, Maas AC, Deckers JW, et al. Early thrombolytic treatment in acute myocardial infarction: reappraisal of the golden hour. Lancet 1996;348:771-5.
2 Reimer KA, Lowe JE, Rasmussen MM, et al. The wavefront phenomenon of ischemic cell death. 1. Myocardial infarct size vs duration of coronary occlusion in dogs. Circulation 1977;56:786-94.

3 Andersen HR, Nielsen TT, Rasmussen K, et al. A comparison of coronary angioplasty with fibrinolytic therapy in acute myocardial infarction. $N$ Engl J Med 2003;349:733-42.

4 Berger PB, Ellis SG, Holmes DR Jr, et al. Relationship between delay in performing direct coronary angioplasty and early clinical outcome in patients with acute myocardial infarction: results from the global use of strategies to open occluded arteries in acute coronary syndromes (GUSTO-Ilb) trial. Circulation 1999; 100:14-20.

5 Nallamothu BK, Bates ER. Percutaneous coronary intervention versus fibrinolytic therapy in acute myocardial infarction: is timing (almost) everything? Am J Cardiol 2003;92:824-6.

6 Antman EM, Anbe DT, Armstrong PW, et al. ACC/AHA guidelines for the management of patients with ST-elevation myocardial infarction--executive summary: a report of the American College of Cardiology/American Heart Association task force on practice guidelines (writing committee to revise the 1999 guidelines for the management of patients with acute myocardial infarction). Circulation 2004;110:588-636.

7 Armstrong PW, Bogaty P, Buller CE, et al. The 2004 ACC/AHA guidelines: a perspective and adaptation for Canada by the Canadian Cardiovascular Society working group. Can J Cardiol 2004;20:1075-9.

8 Widimsky P, Budesinsky T, Vorac D, et al. Long distance transport for primary angioplasty vs immediate thrombolysis in acute myocardial infarction. Final results of the randomized national multicentre trial--PRAGUE-2. Eur Heart $J$ 2003;24:94-104

9 Steg PG, Bonnefoy E, Chabaud S, et al. Impact of time to treatment on mortality after prehospital fibrinolysis or primary angioplasty: data from the CAPTIM randomized clinical trial. Circulation 2003; 108:2851-6.

10 Danchin N, Blanchard D, Steg PG, et al. Impact of prehospital thrombolysis for acute myocardial infarction on 1-year outcome: results from the French Nationwide USIC 2000 Registry. Circulation 2004;110:1909-15.

11 Taher T, Fu Y, Wagner GS, et al. Aborted myocardial infarction in patients with ST-segment elevation: insights from the assessment of the safety and efficacy of a new thrombolytic regimen-3 trial electrocardiographic substudy. J Am Coll Cardiol 2004;44:38-43.

12 Welsh RC, Ornato J, Armstrong PW. Prehospital management of acute STelevation myocardial infarction: a time for reappraisal in North America. Am Heart J 2003;145:1-8.

13 Luepker RV, Raczynski JM, Osganian S, et al. Effect of a community intervention on patient delay and emergency medical service use in acute coronary heart disease: the rapid early action for coronary treatment (REACT) trial. JAMA 2000;284:60-7.

14 Morrison LJ, Verbeek PR, MCDonald AC, et al. Mortality and prehospital thrombolysis for acute myocardial infarction: a meta-analysis. JAMA 2000;283:2686-92.

15 Morrow DA, Antman EM, Sayah A, et al. Evaluation of the time saved by prehospital initiation of reteplase for ST-elevation myocardial infarction: results of the early retavase-thrombolysis in myocardial infarction (ER-TIMI) 19 trial. J Am Coll Cardiol 2002;40:71-7.

16 Bonnefoy E, Lapostolle F, Leizorovicz A, et al. Primary angioplasty versus prehospital fibrinolysis in acute myocardial infarction: a randomised study. Lancet 2002;360:825-9.

17 Wallentin L, Goldstein P, Armstrong PW, et al. Efficacy and safety of tenecteplase in combination with the low-molecular-weight heparin enoxaparin or unfractionated heparin in the prehospital setting: the assessment of the safety and efficacy of a new thrombolytic regimen (ASSENT)-3 plus randomized trial in acute myocardial infarction. Circulation 2003; 108: 135-42.

18 Welsh RC, Goldstein P, Adgey J, et al. Variations in pre-hospital fibrinolysis process of care: insights from the assessment of the safety and efficacy of a new thrombolytic 3 plus international acute myocardial infarction pre-hospital care survey. Eur J Emerg Med 2004;11:134-40.

19 Welsh RC, Gordstein P, Chang WC, et al. Understanding time to treatment in AMI: insights from ASSENT 3 plus. Eur Heart J 2003;24(abstract supp):502.

20 Hirvonen TP, Halinen MO, Kala RA, et al. Delays in thrombolytic therapy for acute myocardial infarction in Finland. Results of a national thrombolytic therapy delay study. Finnish hospitals' thrombolysis survey group. Eur Heart $J$ 1998; 19:885-92.

21 Lambrew CT, Bowlby $\sqcup$, Rogers WJ, et al. Factors influencing the time to thrombolysis in acute myocardial infarction. Time to thrombolysis substudy of the national registry of myocardial infarction-1. Arch Intern Med 1997; 157:2577-82.

22 Goldberg RJ, Steg PG, Sadiq I, et al. Extent of, and factors associated with, delay to hospital presentation in patients with acute coronary disease (the GRACE registry). Am J Cardiol 2002;89(7):791-6.

23 Vaillancourt C, Stiell IG. Cardiac arrest care and emergency medical services in Canada. Can J Cardiol 2004;20:1081-90. 